\title{
Preparing for the coming transnational cancer crisis amid the COVID-19 pandemic
}

\author{
Eric C. Ip ${ }^{1} \cdot$ Shing Fung Lee ${ }^{2}$
}

Received: 7 April 2020 / Accepted: 4 May 2020 / Published online: 29 May 2020

(c) Springer Nature Switzerland AG 2020

\begin{abstract}
The continuing outbreak of the coronavirus disease 2019 (COVID-19) caused by the novel coronavirus SARS-CoV-2 has inflicted considerable burdens onto the health system of China, the world's most populous country. Remarkably, among spectrum of potential mitigation strategies, the Chinese government has implemented all-out lockdowns on large geographical areas, unprecedented in the modern era. This inevitably undermined the right to healthcare of many who now faced great difficulty in getting treatment, especially those with cancer or other life-threatening issues. We elaborate and discuss the medico-legal and human rights consideration triggered by the lockdowns, the unprecedented mass quarantine of Hubei province in China, and the suspension of normal healthcare services. We argue that the same challenge will now be faced by other countries, particularly the USA, Italy, Spain, and France, as the epicentres of COVID-19 has shifted to Europe and the Americas.
\end{abstract}

Keywords Cancer incidence $\cdot$ COVID-19 $\cdot$ Infectious disease $\cdot$ Health policy

It has been estimated that by 2020, China will have over 4.5 million cancer patients and in consequence some 3 million cancer deaths [1]. That number may surge in consequence of the 2019 coronavirus (COVID-19) outbreak, which has been declared a public health emergency of international concern and then a global pandemic by the WHO, as cancer patients have been found to suffer poorer outcomes from COVID19 than those without cancer [2]. Alarmingly, Wuhan, the epicentre of the pandemic, has exhibited mass neglect of treatment of all other patients not infected with COVID19 , including those suffering from cancer. The shortage of medical care for these patients stems from the all-out campaign of the Chinese health authorities to contain COVID19 with drastic lockdown measures at significant social and economic cost. The suspension of care occurs in spite of the mobilization of extra equipment and manpower to Hubei province and its capital Wuhan, and building of makeshift

Shing Fung Lee

leesfm@ha.org.hk

1 Centre for Medical Ethics and Law, The University of Hong Kong, Pokfulam Road, Hong Kong Island, Hong Kong SAR

2 Department of Clinical Oncology, Tuen Mun Hospital, New Territories West Cluster, Hospital Authority, 23 Tsing Chung Koon Road, Tuen Mun, Hong Kong SAR healthcare camps to monitor and care for the 67,794 (as at 15 March 2020) confirmed cases infected with coronavirus, and others suspected to have been infected [3].

The problematic consequences of China's COVID-19 strategy, which has been adopted to varying degrees by many countries, upon the broader healthcare system are beginning to emerge. Many hospitals in Hubei have been designated for and converted to the exclusive care of COVID-19 patients, with previous treatment plans for other patients being postponed or suspended. Additionally, due to fears of COVID19 cross-infection, hospital wards have not been permitted to admit cancer patients and operating theatres have had to be closed. Chemotherapy has been suspended indefinitely. Clinical trials have been similarly affected: participants have been unable to return for follow-up; data collection are no longer timely; many protocols have been violated. Notwithstanding effective measures coping with the needs of cancer patients during the pandemic reportedly adopted by a hospital in Beijing, the nation's capital [5], there is much evidence pointing to a systemic coming crisis in cancer treatment in the world's most populous country, whose magnitude cannot be easily quantified.

People in China with other diseases, even life-threatening ones, are struggling to even get diagnosed, let alone treated. The exclusive public health focus on COVID-19 at the 
expense of even special classes of patients implies that the health system in Hubei, if not elsewhere, is in all likelihood being strained to the breaking point in delivering the usual care while coping with the pandemic, and will remain so for quite some time. Delays in the delivery of oncological care could yield long-lasting, devastating effects at both individual and population levels. Lessons may be learned from the recent history of the Chinese special administrative region of Hong Kong. The former British dependency was one of the jurisdictions most heavily afflicted by the severe acute respiratory syndrome (SARS) outbreak of 2003, suffering almost $40 \%$ of the global death toll [5]. The Hong Kong Cancer Registry recorded a dip in crude cancer incidence to 20,763 during 2003 (the only year to break the uptrend) and a significant rebound the next year [6]. It may have been that the anxiety prevailing in the general public about seeking medical attention caused delayed diagnosis, or the administrative burdens inflicted by the epidemic hampered normal registration processes and then the non-registered cases were carried over to the next year. Note that this happened even though no radical cuts were made to cancer treatment in Hong Kong, as there have been in Hubei this time. When resources become as heavily skewed toward COVID-19 patients as they have in Hubei and elsewhere, the cancer patients currently ignored will face delays in diagnosis and treatment, which will adversely affect oncological outcomes. This very same challenge to healthcare service will be faced by other countries heavily afflicted by the virus such as USA, Italy, Spain, and France as the epicentres of COVID-19 has now shifted to Europe and the Americas.

The Chinese government, as with governments elsewhere, should therefore take steps to prepare for a massive resurgence of cancer patients hitting the healthcare system once the COVID-19 pandemic has been contained. It is understandable that the authorities are under pressure to accord top priority to this containment, especially given the media furore and public anxiety. But it is both ethically and pragmatically dubious that this should be done to the detriment of all other patients. Considerations of justice and of equable resource allocation must not be neglected in national preparedness for cancer incidence [7]. The urgency to reassign priorities so as to give due weight to the needs of cancer patients and ontological research is acute even from a law and policy perspective. China's landmark Basic Healthcare and Health Promotion Law, which codifies a decade of healthcare reforms and is set to enter into force on 1 June 2020, will for the first time guarantee to the Chinese people a positive "right to health", which will in turn obligate the State to enhance citizens' ability to live a "full life cycle" (Article 4), echoing the promises of Article 12 of the International Covenant on Economic, Social and Cultural Rights, signed and ratified by most countries of the world.

Funding None declared.

\section{Compliance with ethical standards}

Conflict of interest None declared.

\section{References}

1. Feng RM, Zong YN, Cao SM (2019) Xu RH (2019) Current cancer situation in China: good or bad news from the 2018 Global Cancer Statistics? Cancer Commun (Lond) 39(1):22. https://doi. org/10.1186/s40880-019-0368-6

2. Liang W, Guan W, Chen R et al (2020) (2020) Cancer patients in SARS-CoV-2 infection: a nationwide analysis in China. Lancet Oncol 21(3):335-337. https://doi.org/10.1016/S1470 -2045(20)30096-6

3. Burki T (2020) Outbreak of coronavirus disease 2019. Lancet Infect Dis 20(292-293):2020. https://doi.org/10.1016/s1473 -3099(20)30076-1

4. World Health Organization (2004) Summary of probable SARS cases with onset of illness from 1 November 2002 to 31 July 2003. https://www.who.int/csr/sars/country/table2004_04_21/ en/. Accessed 15 Mar 2020

5. Wang Z, Wang J, He J (2020) Active and effective measures for the care of patients With cancer during the COVID-19 spread in China. JAMA Oncol. https://doi.org/10.1001/jamao ncol.2020.1198

6. Hospital Authority of Hong Kong (2020) Hong Kong Cancer Registry. https://www3.ha.org.hk/cancereg/. Accessed 15 Mar 2020

7. Rivera YM, Brawley OW (2019) Treating cancer as a public health ethics issue. In: Kahn JP, Kass NE (eds) Mastroianni AC. The Oxford Handbook of Public Health Ethics Oxford University Press, New York

Publisher's Note Springer Nature remains neutral with regard to jurisdictional claims in published maps and institutional affiliations. 